\title{
Mineral Resource Estimation In Grade Control Panel Using Conditional Simulation-Case Study on the Gold Mine
}

\author{
Jinhui Liu ${ }^{1,2}$ a , Xiang Wang ${ }^{3}$, Hancheng Zhang ${ }^{4}$ \\ ${ }^{1}$ Beijing Institute of Geology for Mineral Resources, Beijing, 100012, China \\ 2 Kunming University of Science and Technology, Kunming, 650500, China \\ ${ }^{3}$ Development and Research Center, China Geological Survey, Beijing, 100037, China \\ ${ }^{4}$ Non-ferrous Metals Resource Geological Survey, Beijing, 100012, China
}

\begin{abstract}
In the grade control panel area, when the sampling grid is less than the size of block, the grade is highly smoothed and has very minor variability with the actual "true" value interpolated by Kriging, which is completely different with the actual block grade and tonnage-grade model. In this paper, the optimized the grade distribution and spatial variability were studies by conditional simulation in the Selective Mining Unit (SMU), avoiding the high smoothing effect by linear estimation.
\end{abstract}

\section{INTRODUCTION}

Geostatistics mainly consists of two important fields which are the conditional simulation and kriging. In recent years, the trend of geostatistics study is turning from linear or nonlinear Kriging theory to the conditional simulation. Kriging is mainly used to estimate the global Mineral Resources/ Ore Reserves, but due to the high over smoothing effect, kriging is not suitable for local or recoverable Resource/Ore Reserve estimation. However, conditional simulation has more features on the variability of spatial data with the same statistical characteristics of original data. Therefore, the two different fields represent two different aspects in one same thing, and are not conflicting but complementary.

\section{Principle of Conditional Simulation}

\subsection{Conditional Simulation}

Set $Z(x)$ as the regional variable meeting two order stationarity assumption, $E[Z(x)=m)$, with covariance function $C(h)$ and variogram $\gamma(h)$. To determine $Z(x)$ 's conditional simulation $Z s c(x)$, it is to identify an achievement of isomorphism regional variable $Z s c(x)$ with $Z(x)$ which is equal to the measured value on the measured point $x_{0}$, that is

$$
Z_{S C}(x)=Z\left(x_{0}\right) \quad \forall x_{0}
$$

Here, $Z(x)$ and $Z(x)$ belong to isomorphism, which means they have the same mathematical expectation and

\footnotetext{
a Corresponding author: jhliu0922@qq.com
}

the same distribution histogram, as well as the same $C(h)$ or $\gamma(h)$.

In order to get the equation of conditional simulation $Z_{S C}(x)$, it should use kriging estimate and unconditional simulation $Z_{S}(h)$.

The true value of $Z(x)$ at any point $x$, can be expressed as sum of kriging estimate and its error, that is

$$
Z(x)=Z_{K}^{*}(x)+\left[Z(x)-Z_{K}^{*}(x)\right]=Z_{K}^{*}(x)+R(x)
$$

In which, $R(x)$ is the unknown error in kriging estimate during unconditional simulation. Here kriging error only depends on the data structure and orthogonal to kriging estimate, and then,

$$
E\left\{Z_{K}^{*}(y)\left[Z(x)-Z_{K}^{*}(x)\right]\right\}=0 * x, y
$$

The equation of conditional simulation $Z s c(x)$ can be get by $Z_{S}(x)-Z_{S K}^{*}(x)$ of isomorphism and independent unconditional simulation of kriging error to replace the $Z(x)-Z_{S K}^{*}(x)$ in the Eq. 3, of which $Z s(x)$ is unconditional simulation, and $Z_{S K}^{*}(x)$ is the kriging estimate of $\mathrm{Z}_{S}(x)$.

When considering isomorphism unconditional simulation $Z s(x)$ with $Z(x)$, once getting the achievement of $Z_{S}(x)$ the unconditional simulation value of $Z_{S}\left(x_{a}\right),(a=1,2, \ldots, n)$, can be calculated on the 
observation point $x_{a}$. When kriging is applied to $\left\{Z_{S}\left(x_{a}\right), a=1,2, \ldots, n\right\}$, it will get kriging estimate $Z_{S K}^{*}(x)$ and kriging error $\left[Z(x)-Z_{K}^{*}(x)\right]$. Because $Z_{S}(x)$ and $Z(x)$ are isomorphism, the both Kriging weight coefficient $\lambda_{a}$ are the same, here

$$
Z_{K}^{*}(x)=\sum_{a=1}^{n} \lambda_{a} Z\left(x_{a}\right) ; Z_{S K}^{*}(x)=\sum_{a=1}^{n} \lambda_{a} Z_{S}\left(x_{a}\right)
$$

Further, the kriging error $\left[Z_{S}(x)-Z_{K}^{*}(x)\right]$ and the true kriging error $\left[Z(x)-Z_{K}^{*}(x)\right]$ are isomorphism, therefore $Z_{S}(x)-Z_{S K}^{*}(x)$ can be considered as $\left[Z(x)-Z_{K}^{*}(x)\right]$ 's unconditional simulation. In Eq.3, $\left[Z(x)-Z_{K}^{*}(x)\right]$ and $Z_{S K}^{*}(x)$ are independent, therefore, unconditional simulation of $\left[Z(x)-Z_{K}^{*}(x)\right]$ and $Z_{K}^{*}(x)$ are also independent. $Z_{S}(x)-Z_{S K}^{*}(x)$ can be calculated actually, and also can be replaced the actual kriging error $\left[Z(x)-Z_{K}^{*}(x)\right]$ in Eq. 2. By this time the demined $Z(x)$ is the $Z(x)$ 's conditional simulation value, not $Z(x)$.

$$
Z_{S C}(x)=Z_{K}^{*}(x)+\left[Z_{K}(x)-Z_{S K}^{*}(x)\right]
$$

Both $Z(x)$ and $Z(x)$ have the same variogram, and data structures of kriging estimate $Z_{S K}^{*}(x)$ and $Z_{K}^{*}(x)$ are also the same, therefore the kriging equations are the same, further getting the same solutions of equations and same weight coefficient $\lambda_{a}(a=1,2, \ldots, n)$. Put Eq. 4 into Eq. 5, get,

$$
Z_{S C}(x)=Z_{S}(x)+\sum_{a=1}^{n} \lambda^{a}\left[Z\left(x_{a}\right)-Z_{S}\left(x_{a}\right)\right]
$$

Based on all above, to calculate the conditional simulation $Z_{S C}(x)$ value, first it requires the value of unconditional simulation $Z_{S}(x)$, and then it need carry out the kriging estimate for the difference between the measured values $\left[Z\left(x_{a}\right)-Z_{S}\left(x_{a}\right)\right],(a=1,2, \ldots, n)$ on point $x_{a}$, and then $Z_{S C}(x)$ is equal the both values together.

\subsection{Gaussian anamorphosis}

The Gaussian anamorphosis is a mathematical function which transforms a variable $Y$ with a Gaussian distribution in a new variable $Z$ with any distribution:
$\mathrm{Z}=\varphi(Y)$. For mathematical reasons this function can be conveniently written as a polynomial expansion:

$$
\varphi(Y)=\sum_{i=0}^{\infty} \Psi_{i} H_{i}(Y)
$$

In Eq. 7, Hermite Polynomial is as,

$$
H_{i}(Y)=e^{\frac{y 2}{2}} \frac{d^{i}}{d y^{i}} e^{-\frac{y^{2 i}}{2}}
$$

Hermite polynomial belongs to a special orthogonal polynomial. In practice, this polynomial is divided into several intervals, not with strictly increasing relationship. $\Psi_{i}$ is the expansion coefficient in Hermite Polynomial.

\section{Case Study}

\subsection{Conditional Simulations on Grade Control Panel}

The basic dataset of grade control panel includes horizontal holes, distributing from north to south with different elevation levels $(304,310,313,316,319,322$ and $325 \mathrm{~m})$. Total of 916 holes were drilled, with 7538 assay record. The drilling grid in the long section is of around $33 \times 3 \mathrm{~m}$.

After top_cutting, the basic statistics of the composites samples are that number of composites is 3038, the minimum value is of 0.00 , maximum value is of 74.20 , average grade is of 7.69 , standard deviation is of 6.90 and the variation coefficient is of 0.90 .

The main steps of conditional simulation are summarized as following:

The interval of raw samples was round of $1 \mathrm{~m}$, and all the raw samples were composted to $2 \mathrm{~m}$ to reduce the nugget

Top cutting analysis for the $2 \mathrm{~m}$ composites

Build variogram of the Gaussian transformation

Create 3D grid with SMU size of $5 \times 2 \times 3 \mathrm{~m}$

Conduct the Kriging neighborhood analysis of Gaussian model

Based on the Gaussian model, do 10 numbers of conditional simulation

Fig. 1 shows the histogram of raw Au composites. Fig. 2 gives the Gaussian transformation model after top cutting on $2 \mathrm{~m}$ composites. The basic statistics of Gaussian Au are presented in Fig. 3. Fig. 4 illustrates the experimental varigoram and variogram fitting in three axes by spherical mode.

As expected, the spatial variable $\mathrm{Au}$ has a certain variability characteristics in the grade control domain. The average grade is ranging from $8.27 \mathrm{~g} / \mathrm{t}$ to $8.95 \mathrm{~g} / \mathrm{t}$, at total average grade of $8.68 \mathrm{~g} / \mathrm{t}$ and the total average standard deviation value of around 6.64, as shown in Fig. 5. The plot in the East-West direction is shown in Fig. 6, which shows the grade variability for the 10 sets of 
conditional simulations. Fig. 7, Fig. 8 and Fig. 9 show grade spatial variability on different conditional simulation.

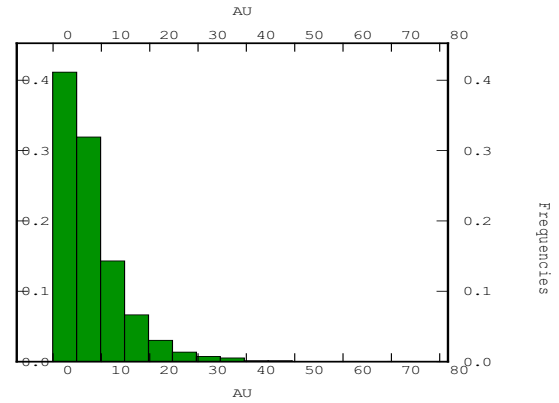

Fig. 1: Histogram of raw $\mathrm{Au}$

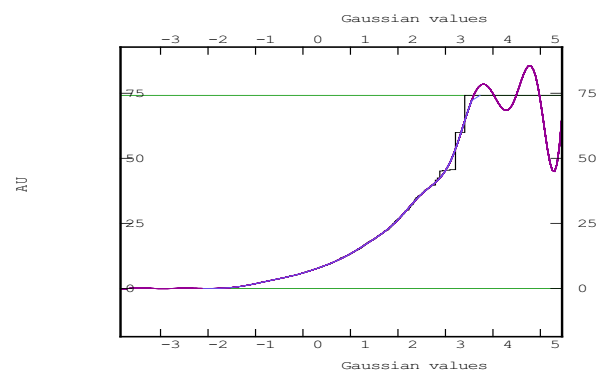

Fig. 2: Au Gaussian transformation model on $2 \mathrm{~m}$ Composites

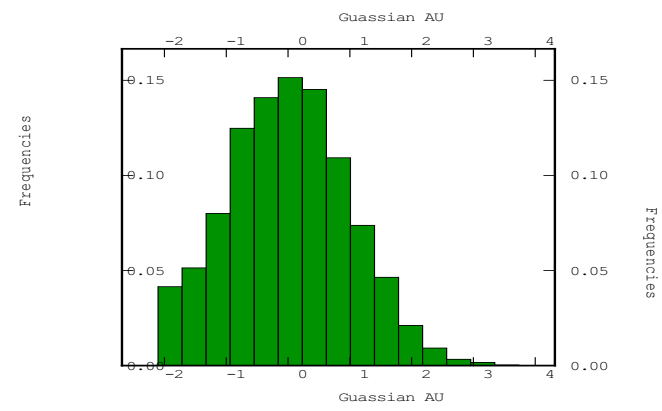

Fig. 3: Histogram of Gaussian $\mathrm{Au}$

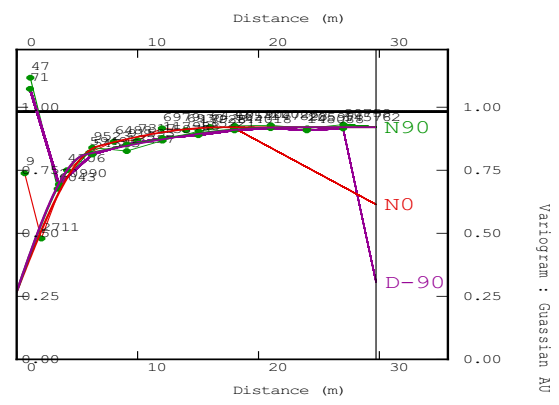

Fig. 4:Vairogram Fitting on Gaussian $\mathrm{Au}$

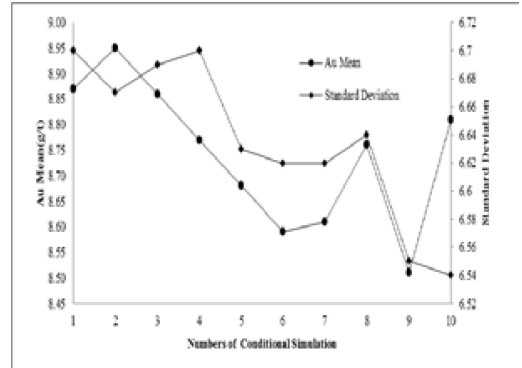

Fig. 5: Au Mean and Standard Deviation in Each Conditional Simulation

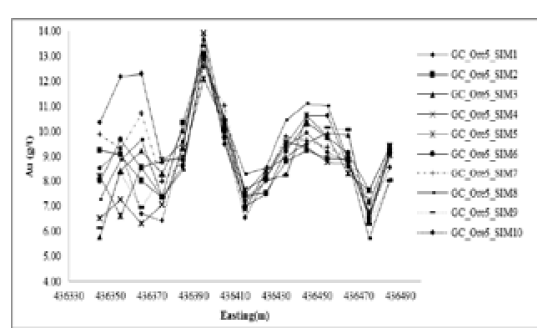

Fig. 6: Grade Trending in East-West Direction for 10 sets of Conditional Simulation

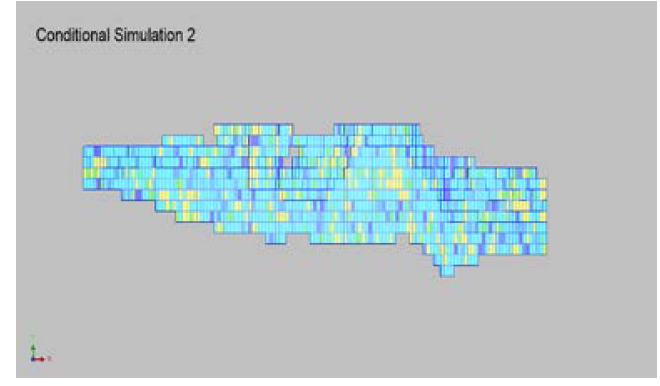

Fig. 7: Au Grade Distribution on Conditional Simulation 2

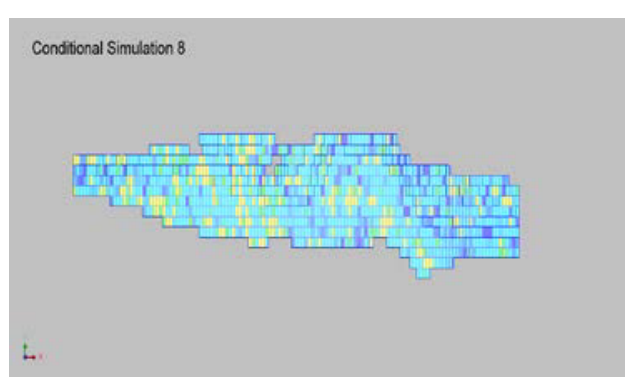

Fig. 8: Au Grade Distribution on Conditional Simulation 8

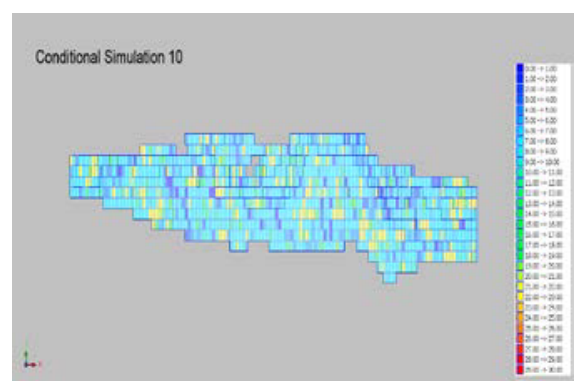

Fig. 9: Au Grade Distribution on Conditional Simulation 10 


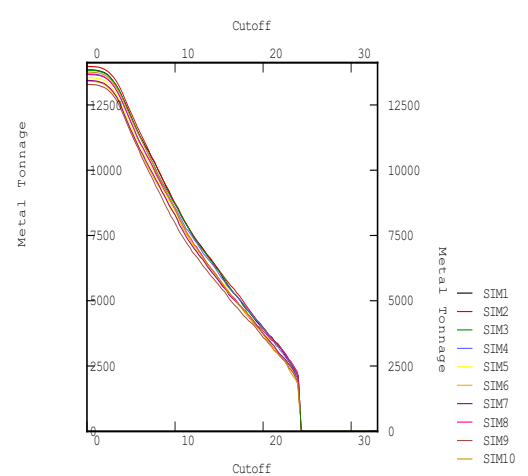

Fig. 10: Grade-Tonnage Curve of Conditional Simulation

\subsection{Resource Estimation Discussion by Conditional simulation}

Theoretically, each simulation value can be used for the resource estimation. The optimized simulation used for the acceptable resource estimation should follow some certain principles, as:

-The simulation values of mean, variation and coefficient of variation are to close with corresponding values of composites

-The simulation variance is twice the estimation variance

- One simulation mean value is the median value of all the simulations

In 10 sets of simulations, simulation 8 was the optimal choice with average grade was 8.76 and standard deviation of 6.64. As shown in Fig. 10, the simulation 8 is located in middle position within most of intervals, which is better used for the resource estimation, avoiding the possible errors caused by the variability. Furthermore, during the mining actives, the conditional simulation can be also used for the mining schedule development and production.

\section{Conclusions}

Due to the highly smoothed effect by kriging, the mineral resource estimation by conditional simulation is in particular irreplaceable in the mining block panel. As for the estimation model, when the sample spacing is less than the actual block size, the interpolated block grade model by kriging may have large differences with the actual mining activities, caused by the high smoothing effect. Therefore, the general linear estimation cannot be used for the actual mining block estimation.

The conditional simulation not only repeats the grade variability in the space, but also the optimized conditional simulation could be used for the resource estimation based on the research of grade, standard deviation and variation. The conditional simulation further can assist arranging the mining production schedule.
1. Royle, A G, 1979. Estimating small blocks of ore, how to do it with confidence, World Mining, April.

2. Stephenson, P R and Vann, J. Commonsense and good communication in mineral resource and ore reserve estimation, in Mineral Resource and Ore Reserve Estimation - The AusIMM Guide to Good Practice, Monograph 23 (Ed: A C Edwards), 2000, pp 13-20 (The Australasian Institute of Mining and Metallurgy: Melbourne).

3. Coombes, J, Thomas, G S, Gifford, M and Jepsen, L. Assessing the risk of incorrect prediction - a nickel/cobalt case study, in Proceedings Mine to Mill,1998, pp 63-68 (The Australasian Institute of Mining and Metallurgy: Melbourne) .

4. Glacken, I M. Change of support and use of economic parameters in block selection, in Geostatistics Wollongong 96 (Eds: E Y Baafi and N A Schofield), 1997, pp 811-821.

5. Nielsen, K I and Currie, D A. The discovery of the Just In Case/Wallaby gold deposit, Laverton District, Western Australia, in Proceedings New Generation Gold Mines '99 Conference, November ,1999, pp 113.

6. Shaw, W J and Khosrowshahi, S.Grade control sampling and ore blocking: Optimization based on Conditional simulation, in Proceedings Third International Mining Geology Conference, 1997, pp131-134 (The Australasian Institute of Mining and Metallurgy Melbourne).

\section{References}

